\title{
Comparison of the ImmuView and the BinaxNOW antigen tests in detection of Streptococcus pneumoniae and Legionella pneumophila in urine
}

\author{
S. Athlin ${ }^{1}$ (I) $\cdot$ A. Iversen ${ }^{2,3} \cdot$ V. Özenci ${ }^{2,4}$
}

Received: 4 April 2017 / Accepted: 12 May 2017 /Published online: 6 June 2017

(C) The Author(s) 2017. This article is an open access publication

\begin{abstract}
The use of urinary antigen tests (UATs) may provide early etiology in pneumonia, and facilitates rapid and directed antibiotic treatment. In this study, we evaluated the novel lateral flow ImmuView Streptococcus pneumoniae and Legionella pneumophila UAT, which detects pneumococcal and L. pneumophila serogroup 1 antigens in a combined test. We compared the ImmuView UAT with the BinaxNOW S. pneumoniae UAT and the BinaxNOW L. pneumophila UAT in 147 patients with pneumococcal bacteremia ( $n=48)$, non-pneumococcal non-Legionella bacteremia $(n=93)$ and Legionella infections in the lower airways (L. pneumophila, $n=5 ;$ L. bozemanii, $n=1$ ). In three cases, the ImmuView test was invalid before and after boiling while the BinaxNOW tests were valid in all cases. In 144 cases, the three UATs demonstrated a very good inter-assay agreement for detection of pneumococcal antigen $(\kappa=0.86)$ and L. pneumophila antigen $(\kappa=1.00)$. The ImmuView and BinaxNOW S. pneumoniae tests had similar sensitivities ( $62 \%$ vs $60 \%$; $p=\mathrm{ns}$ ) in 48 cases with pneumococcal bacteremia and both tests had specificities of $97 \%$ in 96 cases with
\end{abstract}

S. Athlin

simon.athlin@ regionorebrolan.se

1 Department of Infectious Diseases, Faculty of Medicin and Health, Örebro University, S-701 85 Örebro, Sweden

2 Division of Clinical Microbiology, Department of Laboratory Medicine, Karolinska Institutet, Stockholm, Sweden

3 Department of Clinical Microbiology, Karolinska University Hospital, Solna, Stockholm, Sweden

4 Department of Clinical Microbiology F 72, Karolinska Institutet, Karolinska University Hospital, SE 14186 Huddinge, Stockholm, Sweden non-pneumococcal infections. Furthermore, the ImmuView and BinaxNOW L. pneumophila tests were positive for Legionella antigen in five patients with confirmed L. pneumophila serogroup 1 infections, and negative in all non- $L$. pneumophila cases. The ImmuView and BinaxNOW tests performed similarly when evaluated on urine samples from bacteremic and non-bacteremic patients with identified etiology.

\section{Introduction}

The use of urinary antigen tests (UATs) is recommended in hospitalized patients with community-acquired pneumonia to support early and directed antibiotic therapy [1,2]. Antigen detection in urine has many advantages, since urine is excreted in large quantities and collection does not require invasive methods [3]. In previous studies, a positive UAT result was supportive for the clinician to narrow the antibiotic treatment [4-6]. However, in a recent study by Falguera et al., detection of Streptococcus pneumoniae and Legionella pneumophila antigens by UATs did not associate with improved patient outcome or cost-effectiveness [7]. By the development of novel UATs, with enhanced performance of detecting these pathogens, the clinical and economic benefits by using UATs may be improved.

Several monovalent UATs are available for detection of $S$. pneumoniae and L. pneumophila polysaccharides by separate tests $[3,8,9]$. Recently, the combined lateral flow test, the ImmuView ${ }^{\circledR}$ S. pneumoniae and L. pneumophila (Statens Serum Institut Diagnostica (SSID), Denmark) UAT was introduced on the market. The test is based on immunochromatographic technology for detection of $S$. pneumoniae and L. pneumophila serogroup 1 antigens and is the first UAT that detects both pathogens in a single test. 
According to Jørgensen, Uldum (both Statens Serum Institut, Denmark) and co-authors (SSID), the combined sensitivity of the ImmuView test for detection of S. pneumoniae and L. pneumophila antigens was higher than of three competing tests in combination ( $87 \%$ vs $74 \%$ ) [10]. The specificity of the test showed high rates $(100 \%$ for L. pneumophila serogroup 1 and $99 \%$ for $S$. pneumoniae) but did not include bacteremic patients with non-S. pneumoniae non-Legionella etiology. False-positive UAT results have been observed in patients with positive blood cultures (BC) for $\alpha$-hemolytic streptococci and Gram-negative bacteria previously [11, 12]. Therefore, the analyses of both sensitivity and specificity have utmost clinical relevance for targeting antibiotic treatment according to UAT results. In this study, we aimed to evaluate the performance of the ImmuView test on bacteremic patients with identified pneumococcal and non-pneumococcal etiology, as well as on patients with Legionnaires' disease.

\section{Materials and methods}

Urine samples from adult patients $(\geq 18$ years $)$ with community-acquired infections were collected between September 2013 and October 2016 at Örebro University Hospital, Karolinska University Hospital Huddinge, and Skåne University Hospital Malmö. Inclusion criteria were urine samples from (i) bacteremic patients with simultaneous blood culture positivity or (ii) non-bacteremic patients with lower respiratory tract samples containing Legionella spp. Culture on blood and urine was performed by standard methods. One sample per patient was analyzed. Cases with coagulase-negative staphylococci in only one $\mathrm{BC}$ bottle were excluded. Isolated bacteria from $\mathrm{BC}$ were identified to species level according to standard methods. L. pneumophila cases were identified by PCR on BAL or sputum and confirmed by Binax ${ }^{\circledR}$ L. pneumophila serogroup 1 enzyme immunoassay (EIA; Alere, USA) on urine. One L. bozemanii case was identified by culture and PCR for Legionella spp. on BAL and sputum and the isolate was determined on species level by matrix-assisted laser desorption ionization-time of flight mass spectrometry (MALDI Biotyper®; Bruker Daltonics, USA). All clinical samples, along with standard microbiological test results, age and gender were anonymized before storage at $-20{ }^{\circ} \mathrm{C}$.

All urine samples were thawed in room temperature for a blinded and simultaneous testing procedure with the ImmuView S. pneumoniae and L. pneumophila test, the BinaxNOW S. pneumoniae test, and the BinaxNOW L. pneumophila test. The ImmuView test is a combined lateral flow test based on immunochromatographic technology with one control line for validation and two test lines for detection of S. pneumoniae and L. pneumophila serogroup1 antigens, respectively. The test was performed according to the manufacturer's instructions, as three drops of urine and two drops of running buffer were added to a test tube and gently whirled. The test strip was then inserted into the tube, and test results were read after $15 \mathrm{~min}$. Any visible pink/red (S. pneumoniae) or blue (L. pneumophila) test line was considered as a positive result. A test result was considered invalid if the control line was absent or if the test showed three gray/purple test lines. The samples were vortexed for $5 \mathrm{~s}$ but not boiled or concentrated before the first testing procedure. Any urine sample with discrepant or invalid test result was retested, as well as all urine samples with a positive result for L. pneumophila serogroup 1 . The re-testing procedure was done before boiling and after boiling for $10 \mathrm{~min}$. Invalid tests were examined regarding levels of leucocytes, erythrocytes, and glucose levels by Multistix ${ }^{\circledR} 7$ (Siemens Healthcare Diagnostics, USA).

The inter-assay agreement between the tests was estimated by calculating Cohen's unweighted kappa coefficient $(\kappa)$. McNemar's test was used for the comparison of sensitivity and specificity rates. A confidence interval (CI) of $95 \%$ was used for statistical precision. A two-tailed $p$-value of $<0.05$ was considered statistically significant. The statistical analyses were performed with a statistical software package (SPSS for Windows, version 17.0).

\section{Results}

Of 147 cases included in this study, 141 were bacteremic cases (median age, 74 years (range, 18-96 years); female, 44\%) with isolated bacteria from $\mathrm{BC}$ as shown in Table 1. In addition, six non-bacteremic cases with lower respiratory tract samples containing $L$. pneumophila serogroup $1[n=5$; median age, 57 years (range, 52-82 years); all males] and Legionella bozemanii ( $n=1$; age, 42 years; male) were included.

Table 2 shows the test results of the three UATs obtained by category of infection. In three cases $(2.1 \%)$, the ImmuView tests were invalid since no control lines were observed. In one case (E. coli in $\mathrm{BC})$, the test line for pneumococcal antigen turned gray, and no other lines were visible at the initial testing as well as after boiling. In the second case (E. coli in BC), the test line for pneumococcal antigen turned gray, and no other lines were visible but all three lines turned gray after boiling. In the third case (P. mirabilis in $\mathrm{BC}$ ), no lines were visible at the initial testing or after boiling. In the first case, the urine sample contained a high level of glucose $(28 \mathrm{mmol} / \mathrm{l})$ and all three cases showed elevated levels of leucocytes $(>70$ leucocytes $/ \mu \mathrm{l})$ and erythrocytes ( $>80$ erythrocytes $/ \mu \mathrm{l})$. The third sample was highly viscous before as well as after boiling. In these three samples, the BinaxNOW tests were valid and the results were negative. 
Table 1 Blood culture isolates of 141 bacteremic patients in the study

Blood culture isolate No. of patients

\begin{tabular}{ll}
\hline Streptococcus pneumoniae & 48 \\
Escherichia coli & 28 \\
Staphylococcus aureus & 12 \\
Enterococcus faecalis & 8 \\
Staphylococcus epidermidis & 5 \\
Klebsiella pneumoniae & 4 \\
Proteus mirabilis & 4 \\
Pseudomonas aeruginosa & 3 \\
Streptococcus mitis & 3 \\
Klebsiella oxytoca & 2 \\
Other* & 24 \\
\hline
\end{tabular}

*One each of Bacillus cereus, Bacteroides aerogenes, Bacteroides caccae, Bacteroides fragilis, Clostridium paraputrificum, Enterococcus cloacae, Granulicatella adiacens, Group G streptococcus, Listeria monocytogenes, Morganella morganii, Salmonella typhimur, Streptococcus agalactiae, Streptococcus anginosus, Streptococcus bovis, Streptococcus pyogenes, Streptococcus salivarius, Clostridium perfringens + E. coli, E. faecalis $+\alpha$-hemolytic streptococcus, E. faecalis + P. mirabilis, E. faecalis $+S$. agalactiae, E. faecium + $P$. aeruginosa, $C$. perfringens $+E$. faecium $+S$. epidermidis, $C$. perfringens $+K$. pneumoniae $+S$. anginosus and $S$. epidermidis + S. salivarius $+S$. mitis

Calculated on 144 of 147 cases (98\%) with valid test results, the inter-assay agreement between the ImmuView test and the BinaxNOW S. pneumoniae test for detection of pneumococcal antigen was very good ( $\kappa=0.86$; CI, 0.76-0.96). Both tests were positive in 29 cases and negative in 108 cases, and with discrepant results in seven cases of pneumococcal bacteremia (Table 2). Discrepant results were confirmed by retesting. The inter-assay agreement between the ImmuView test and the BinaxNOW L. pneumophila test for detection of L. pneumophila serogroup 1 antigen was very good $(\kappa=1.00$; CI, 1.00-1.00); both tests were positive in five cases and negative in 139 cases.

The performance rates were calculated on cases with valid test results for all three tests. For detection of pneumococcal antigen in urine, the sensitivity in patients with pneumococcal bacteremia was $62 \%$ (30/48) for the ImmuView test and $60 \%$ $(29 / 48 ; p=n s)$ for the BinaxNOW $S$. pneumoniae test. Calculated on non-pneumococcal cases (bacteremic, $n=90$; non-bacteremic Legionella spp., $n=6$ ), both tests yielded specificity rates of $97 \%$ (93/96), since both tests showed false-positive results in three cases with non-pneumococcal bacteremia (E. coli, E. faecalis and S. epidermidis). The false-positive test results were confirmed by re-testing the samples.

The ImmuView and the BinaxNOW L. pneumophila tests were positive for Legionella antigen in all cases $(5 / 5,100 \%)$ with L. pneumophila serogroup 1 etiology, and the tests were positive at re-testing before and after boiling for $10 \mathrm{~min}$. Furthermore, both tests were negative in all cases (139/139; $100 \%$ ) with non-L. pneumophila etiology, including the L. bozemanii case.

\section{Discussion}

The ImmuView S. pneumoniae and L. pneumophila UAT may provide benefits in terms of reduced laboratory work due to the detection of both pathogens in a single test. However, the combination of two assays is of concern, since the duality of the test may influence the test performance negatively. In this study, the ImmuView test showed a very good inter-assay
Table 2 Test results of the ImmuView S. pneumoniae and L. pneumophila $\mathrm{UAT}$ in comparison with the BinaxNOW S. pneumoniae UAT and the BinaxNOW L. pneumophila UATs in 147 bacteremic and nonbacteremic patients

\begin{tabular}{|c|c|c|c|c|c|}
\hline \multirow[b]{2}{*}{ Category of infection } & \multirow[b]{2}{*}{$\begin{array}{l}\text { No. } \\
\quad \text { tested } \\
(n=147)\end{array}$} & \multicolumn{2}{|l|}{ ImmuView UAT } & \multicolumn{2}{|c|}{ BinaxNOW UATs } \\
\hline & & $\begin{array}{l}\text { No. positive } \\
\quad(\%) \\
\text { S. pneumoniae }\end{array}$ & $\begin{array}{l}\text { No. positive } \\
\text { L. pneumophila }\end{array}$ & $\begin{array}{l}\text { No. positive } \\
\quad(\%) \\
\text { S. } \\
\quad \text { pneumon- } \\
\quad \text { iae }\end{array}$ & $\begin{array}{l}\text { No. positive } \\
\text { L. pneumophila }\end{array}$ \\
\hline $\begin{array}{l}\text { Bacteremic, } \\
\text { pneumococcal }\end{array}$ & 48 & $30 / 48^{\mathrm{a}}(62)$ & 0 & $29 / 48^{\mathrm{a}}(60)$ & 0 \\
\hline $\begin{array}{l}\text { Bacteremic, } \\
\text { non-pneumococcal }\end{array}$ & 93 & $3 / 93^{\mathrm{b}}(3)$ & 0 & $3 / 93(3)$ & 0 \\
\hline Non-bacteremic & 5 & 0 & $5 / 5$ & 0 & $5 / 5$ \\
\hline L. pneumophila & & & & & \\
\hline $\begin{array}{l}\text { Non-bacteremic } \\
\text { L. bozemanii }\end{array}$ & 1 & 0 & 0 & 0 & 0 \\
\hline
\end{tabular}

UAT urinary antigen test

${ }^{a}$ In 26 of 48 pneumococcal bacteremia cases, the ImmuView and the BinaxNOW S. pneumoniae UATs showed concordant positive test results. In seven cases, the UATs showed discrepant results

${ }^{\mathrm{b}}$ The ImmuView UAT showed valid test results in 90 of 93 non-pneumococcal bacteremia cases, three of which showed positive test results 
agreement in comparison with the BinaxNOW S. pneumoniae UAT and the BinaxNOW L. pneumophila UAT in infected patients with identified etiology. Thus, our study showed that the ImmuView test performed similarly to the BinaxNOW tests in bacteremic and non-bacteremic patients.

For detection of pneumococcal antigen in bacteremic patients, the sensitivities were similar of the ImmuView and the BinaxNOW S. pneumoniae tests ( $62 \%$ vs $60 \% ; p=n s)$. In comparison, sensitivities of the BinaxNOW test ranged between 63 and $88 \%$ in bacteremic patients in previous studies [9]. In the study by Jørgensen et al., the sensitivity was $84 \%$ for the ImmuView test and $78 \%$ for the BinaxNOW test ( $p=\mathrm{ns}$ ) on un-boiled urine samples from bacteremic patients [10]. In addition, the ImmuView was recently compared against the BinaxNOW $S$. pneumoniae test and two L. pneumophila EIAs, as described by Lindsay et al. [13]. The test was positive in 70/78 (valid test results, 90\%) urine samples from previously Legionella positive patients and was positive for pneumococcal antigen in $45 / 554$ (8\%) patients with undefined status of infection.

Cross-reactivity between S. pneumoniae and other bacterial species may yield false-positive results in patients with nonpneumococcal infections. In the present study, the ImmuView and the BinaxNOW S. pneumoniae tests were both positive for pneumococcal antigens in three bacteremic cases with non-pneumococcal etiology, yielding specificities of $97 \%$ for both tests (Table 2). Similarly, Smith et al. found that the BinaxNOW test was positive in 3/106 adult patients with community-acquired septic infections caused by E. coli, K. pneumoniae and Enterobacter spp. in BC, yielding a specificity of 97\% [11]. When Jørgensen et al. evaluated the specificity of the ImmuView test in 76 non-bacteremic patients with urinary tract infections, the test was negative for pneumococcal antigen in all but one case (specificity, 99\%), while the BinaxNOW test was negative in all cases [10]. Furthermore, they noted that the test was positive for both pneumococcal and Legionella antigens in 4/71 (6\%) cases with pneumococcal bacteremia, which was considered as either double infections or due to false-positivity [14]. Dualpositivity of the ImmuView test was also observed in $8 / 45$ patients $(18 \%)$ with unknown etiology in the study by Lindsay et al. [13], but in the present study no dualpositivity was observed.

Previously, when we evaluated the Uni-Gold $S$. pneumoniae UAT on bacteremic patients, we observed false-positive test results in $15 \%(7 / 48)$ of patients with E. coli and in $32 \%(6 / 19)$ of patients with $\alpha$-hemolytic streptococcus etiology respectively [12]. The Uni-Gold test was developed by the same manufacturer as the ImmuView test (SSID, Denmark) and was based on similar lateral flow immunochromatographic technology for detection of pneumococcal antigen. However, in the present study, we noted false-positive results for the detection of pneumococcal antigen in only one case of 39 (3\%) of E. coli bacteremia, and in no case of $\alpha$-hemolytic streptococcus bacteremia $(n=9)$. This may indicate that the ImmuView and the UniGold tests actually differ in specificity, presumably due to variations in test technologies.

For detection of L. pneumophila serogroup 1 antigen in urine, the ImmuView test and the BinaxNOW L. pneumophila test performed similarly, yielding positive results in all five $L$. pneumophila serogroup 1 cases and negative in all other cases, including one L. bozemanii case. Calculated on this limited set of samples, the sensitivity and the specificity rates yielded $100 \%$ for each test. In comparison, in a metaanalysis by Shimada et al., the pooled sensitivity and specificity rates for the BinaxNOW L. pneumophila test were $90 \%$ and 99\% respectively [8]. Jørgensen et al. reported sensitivities and specificities in patients with urinary tract infections of $87 \%$ and $100 \%$ for the ImmuView test, and $78 \%$ and $100 \%$ for the BinaxNOW test respectively [10]. However, in 2/55 (4\%) of confirmed L. pneumophila serogroup 1 cases, the test was negative for Legionella but positive for S. pneumoniae, which was interpreted as either double infection or false-positivity as described above.

We used PCR on lower respiratory samples for identification of Legionella cases, which is suggested to be a reliable method for the diagnosis of Legionnaires' disease [15, 16]. PCR is more sensitive than airway culture and UAT for detection of Legionella spp., which may be accredited to a high detection rate at a low bacterial load [17, 18]. Moreover, we used the Binax L. pneumophila serogroup 1 EIA to determine the serogroup of PCR positive L. pneumophila cases. The sensitivity of this assay has been demonstrated to be moderate (74\%), but with high specificity (99\%), and was used as a reference method to evaluate the BinaxNOW L. pneumophila test previously $[10,19]$. Accordingly, we consider the positive results by the ImmuView and BinaxNOW in our study to be reliable, as we used the combined reference standard of PCR and EIA. However, due to the low number of cases with $L$. pneumophila serogroup 1 etiology and the fact that the cases were not determined on a subgroup level, we may have overlooked potential differences in performance between the tests, and further analysis with larger group of patients are warranted.

In three cases, the ImmuView tests were invalid while the BinaxNOW tests were valid in all cases. High viscosity was the presumable reason for invalidity in the first case, but in the two other cases the cause of invalidity remains unknown. In the study by Lindsay et al., the ImmuView showed invalid test results when applied on 7/554 retrospectively collected and frozen urine samples (1\%) [13]. In five cases, the samples derived from patients with previously positive culture, serology, or Binax EIA for L. pneumoniae but no other information of the samples or the test lines are available. In the study by Jørgensen et al., all urine samples were frozen at $-20^{\circ} \mathrm{C}$, and 
showed valid test results after they had been thawed. Evaluation studies of UATs are often performed on frozen urine samples, since pneumococcal and Legionella polysaccharides are considered to be temperature-stable molecules for moderate time periods $[10,20,21]$.

However, the impact of long-term freezing may influence EIA results negatively [22]. When we investigated a 15-yearold urine sample (not included in the study) collected from a patient positive for L. pneumophila by culture and PCR, the Binax L. pneumophila serogroup 1 EIA was negative, with a mean absorbance ratio of 1.8 in comparison to the negative control (positive reference ratio, >3.0). Low antigen concentration or long-term freezing may have affected the result negatively but was not further investigated. In addition, the ImmuView test was negative but the Binax NOW L. pneumoniae test was faintly positive when the UATs were applied to the sample. This discrepancy of test results may be due to a higher sensitivity of the BinaxNOW test or a falsepositive result on a non-serogroup 1 sample, but it has been recommended previously that very weak positive lines should be interpreted cautiously [19]. In our study, we believe that the comparison between the tests was not negatively affected by freezing of the samples, since all samples were frozen for a moderate time period and were tested simultaneously, but we recommend further studies on unfrozen samples.

In conclusion, the sensitivity and specificity of the ImmuView test and the BinaxNOW tests were similar when evaluated on urine samples from infected patients with identified etiology. No difference in specificities between the tests was observed in bacteremic patients with non-pneumococcal non-Legionella etiology.

Acknowledgements We thank Anna Nilsson at the Department of Infectious Diseases, Lunds University and Thikra Salim Ashak at the Department of Clinical Microbiology, Karolinska University Hospital Huddinge for assistance in collecting clinical samples.

\section{Compliance with ethical standards}

Funding The study was supported by grants from the Region Örebro County Council Research Committee (OLL-524181). The ImmuView tests were provided by SSI Diagnostica, Hillerød, Denmark and the BinaxNOW tests were provided by Alere International Ltd., Galway, Ireland, respectively.

Conflict of interest The authors declare that they have no conflict of interest.

Ethical standards This study was performed in accordance with the Declaration of Helsinki and with the ethical standards of the research committees in Sweden.

Informed consent Not applicable.

Open Access This article is distributed under the terms of the Creative Commons Attribution 4.0 International License (http:// creativecommons.org/licenses/by/4.0/), which permits unrestricted use, distribution, and reproduction in any medium, provided you give appropriate credit to the original author(s) and the source, provide a link to the Creative Commons license, and indicate if changes were made.

\section{References}

1. Mandell LA, Wunderink RG, Anzueto A, Bartlett JG, Campbell GD, Dean NC, Dowell SF, File TM Jr, Musher DM, Niederman MS, Torres A, Whitney CG (2007) Infectious Diseases Society of America/American Thoracic Society consensus guidelines on the management of community-acquired pneumonia in adults. Clin Infect Dis 44(Suppl 2):S27-S72. doi:10.1086/511159

2. Lim WS, Baudouin SV, George RC, Hill AT, Jamieson C, Le Jeune I, Macfarlane JT, Read RC, Roberts HJ, Levy ML, Wani M, Woodhead MA (2009) BTS guidelines for the management of community acquired pneumonia in adults: update 2009. Thorax 64(Suppl 3):iii1-iii55. doi:10.1136/thx.2009.121434

3. Tuuminen $\mathrm{T}$ (2012) Urine as a specimen to diagnose infections in twenty-first century: focus on analytical accuracy. Front Immunol 3:45. doi:10.3389/fimmu.2012.00045

4. Sorde R, Falco V, Lowak M, Domingo E, Ferrer A, Burgos J, Puig M, Cabral E, Len O, Pahissa A (2011) Current and potential usefulness of pneumococcal urinary antigen detection in hospitalized patients with community-acquired pneumonia to guide antimicrobial therapy. Arch Intern Med 171(2):166-172. doi:10.1001/ archinternmed.2010.347

5. Guchev IA, Yu VL, Sinopalnikov A, Klochkov OI, Kozlov RS, Stratchounski LS (2005) Management of nonsevere pneumonia in military trainees with the urinary antigen test for Streptococcus Pneumoniae: an innovative approach to targeted therapy. Clin Infect Dis 40(11):1608-1616. doi:10.1086/429919

6. Yu VL (2011) A clinical solution to antimicrobial resistance in community-acquired pneumonia: narrowing the spectrum of antimicrobial therapy: comment on "current and potential usefulness of pneumococcal urinary antigen detection in hospitalized patients with community-acquired pneumonia to guide antimicrobial therapy". Arch Intern Med 171(2):172-173. doi:10.1001/archinternmed. 2010.474

7. Falguera M, Ruiz-Gonzalez A, Schoenenberger JA, Touzon C, Gazquez I, Galindo C, Porcel JM (2010) Prospective, randomised study to compare empirical treatment versus targeted treatment on the basis of the urine antigen results in hospitalised patients with community-acquired pneumonia. Thorax 65(2):101-106. doi:10. 1136/thx.2009.118588

8. Shimada T, Noguchi Y, Jackson JL, Miyashita J, Hayashino Y, Kamiya T, Yamazaki S, Matsumura T, Fukuhara S (2009) Systematic review and metaanalysis: urinary antigen tests for Legionellosis. Chest 136(6):1576-1585. doi:10.1378/chest.082602

9. Sinclair A, Xie X, Teltscher M, Dendukuri N (2013) Systematic review and meta-analysis of a urine-based pneumococcal antigen test for diagnosis of community-acquired pneumonia caused by Streptococcus Pneumoniae. J Clin Microbiol 51(7):2303-2310. doi: $10.1128 / \mathrm{jcm} .00137-13$

10. Jorgensen CS, Uldum SA, Sorensen JF, Skovsted IC, Otte S, Elverdal PL (2015) Evaluation of a new lateral flow test for detection of Streptococcus Pneumoniae and Legionella pneumophila urinary antigen. J Microbiol Methods 116:33-36. doi:10.1016/j. mimet.2015.06.014

11. Smith MD, Derrington P, Evans R, Creek M, Morris R, Dance DAB, Cartwright K (2003) Rapid diagnosis of Bacteremic 
pneumococcal infections in adults by using the Binax NOW Streptococcus Pneumoniae urinary antigen test: a prospective, controlled clinical evaluation. J Clin Microbiol 41(7):2810-2813. doi: 10.1128/jcm.41.7.2810-2813.2003

12. Athlin S, Altun O, Eriksen HB, Ozenci V, Stralin K (2015) The Uni-Gold Streptococcus Pneumoniae urinary antigen test: an interassay comparison with the BinaxNOW(R) Streptococcus Pneumoniae test on consecutive urine samples and evaluation on patients with bacteremia. Eur J Clin Microbiol Infect Dis 34(8): 1583-1588. doi:10.1007/s10096-015-2390-1

14. Tan MJ, Tan JS, File TM Jr (2002) Legionnaires disease with bacteremic coinfection. Clin Infect Dis 35(5):533-539. doi:10.1086/ 341771

13. Lindsay D, Scott K, McNeill A, Rankin S, Smith A (2016) Evaluation of the rapid lateral flow ImmuView ${ }^{\circledR}$ S. Pneumoniae and L. pneumophila urinary antigen test (UAT) for the detection of antigens in urine. Paper presented at the 10th International Symposium On Pneumococci and Pneumococcal Diseases, Glasgow, UK

15. Avni T, Bieber A (2016) Diagnostic accuracy of PCR alone and compared to urinary antigen testing for detection of Legionella spp.: a. Systematic Review 54(2):401-411. doi:10.1128/jcm.02675-15

16. Peci A, Winter AL, Gubbay JB (2016) Evaluation and comparison of multiple test methods, including real-time PCR, for Legionella detection in clinical specimens. Front Public Health 4:175. doi:10. 3389/fpubh.2016.00175
17. Murdoch DR, Podmore RG, Anderson TP, Barratt K, Maze MJ, French KE, Young SA, Chambers ST, Werno AM (2013) Impact of routine systematic polymerase chain reaction testing on case finding for Legionnaires' disease: a pre-post comparison study. Clin Infect Dis 57(9):1275-1281. doi:10.1093/cid/cit504

18. Diederen BM (2008) Legionella spp. and Legionnaires' disease. J Infect 56(1):1-12. doi:10.1016/j.jinf.2007.09.010

19. Helbig JH, Uldum SA, Luck PC, Harrison TG (2001) Detection of Legionella pneumophila antigen in urine samples by the BinaxNOW immunochromatographic assay and comparison with both Binax Legionella urinary enzyme immunoassay (EIA) and Biotest Legionella urin antigen EIA. J Med Microbiol 50(6):509516. doi:10.1099/0022-1317-50-6-509

20. Guerrero C, Toldos CM, Yague G, Ramirez C, Rodriguez T, Segovia M (2004) Comparison of diagnostic sensitivities of three assays (Bartels enzyme immunoassay [EIA], Biotest EIA, and Binax NOW immunochromatographic test) for detection of Legionella pneumophila serogroup 1 antigen in urine. J Clin Microbiol 42(1):467-468

21. Dominguez J, Gali N, Blanco S, Pedroso P, Prat C, Matas L, Ausina V (2001) Detection of Streptococcus Pneumoniae antigen by a rapid immunochromatographic assay in urine samples. Chest 119(1):243-249

22. Chang FY, Stout JE, Yu VL (1996) Assessment of enzyme immunoassay versus radioimmunoassay for detection of Legionella pneumophila serogroup 1 antigen in frozen urine specimens. J Clin Microbiol 34(10):2628-2629 